\title{
Treatment recommendations for cystic fibrosis-related diabetes: too little, too late?
}

\section{Brandon Nathan, Antoinette Moran}

In the general population, altered glucose metabolism is associated with deleterious microvascular changes, inflammatory cardiovascular disease and death. Large population studies have established clinically relevant glucose threshold levels associated with these risks. These have led to the currently accepted definitions of diabetes and oral glucose tolerance abnormalities as well as recommendations for treatment. While these same definitions and treatment recommendations may be appropriate to prevent microvascular disease in those with cystic fibrosis (CF), they may be inadequate when it comes to preventing the major complications of diabetes in $\mathrm{CF}$-namely, respiratory failure and death.

CF-related diabetes (CFRD) is primarily caused by insulin insufficiency as a result of pancreatic fibrosis and islet destruction. In addition, there is a variable component of insulin resistance related to underlying chronic inflammation superimposed with bouts of acute infection. Patients with CFRD are at risk of typical diabetic microvascular complications which, although they occur less frequently and may be less severe than in other diabetes populations, are similarly related to duration of diabetes and the level of glycaemic control. ${ }^{1}$ In contrast, macrovascular disease-the primary cause of mortality in the general diabetes population-is not known to occur in CFRD, perhaps at least in part because cholesterol levels are low. However, CFRD is associated with its own unique morbidity and mortality profile which has been explored in detail in a recent technical review. ${ }^{2}$

A number of studies have documented worse survival in CF patients with diabetes than in those without. As in

Department of Pediatrics, University of Minnesota Amplatz Children's Hospital, Minneapolis, Minnesota, USA

Correspondence to Brandon Nathan, Pediatric Endocrinology, University of Minnesota, 13-124 PWB, MMC 404, 516 Delaware St. SE, Minneapolis, MN 55455, USA; natha039@umn.edu most people with CF, death is due to chronic inflammatory lung disease, which appears to be exacerbated by CFRD. This may be related to the catabolic effects of insulin insufficiency leading to excessive protein and lipid breakdown. Normal body weight and lean body mass, known to be critical for preservation of CF lung function, are difficult for these patients to achieve and maintain in a catabolic environment. In addition to the deleterious effects of insulin insufficiency, there is increasing evidence that hyperglycaemia per se has a negative impact on lung function by creating a pro-inflammatory pro-bacteria environment, including the elevation of glucose levels in airway surface liquid. Fortunately, clinical studies have shown that aggressive institution of insulin replacement therapy improves weight, pulmonary function and survival in CFRD. ${ }^{3}$ It has been clearly established that patients with even mild diabetes (CFRD without fasting hyperglycaemia) benefit from early insulin therapy. ${ }^{4}$

What is still not clear is whether patients with less severe glucose tolerance abnormalities would benefit from treatment typically associated with diabetes. Because of pancreatic damage, few patients with CF have truly normal glucose tolerance. Although the oral glucose tolerance test (OGTT) remains the gold standard for screening and characterising this population, the traditional interpretation of the test (ie, baseline and $2 \mathrm{~h}$ glucose levels) misses mid-OGTT abnormalities. Other screening strategies, including the use of continuous glucose monitors or additional interpretations of OGTT data such as glucose or insulin area under the curve (AUC) or assessment of glucose levels at mid-OGTT time points, may eventually identify a subset of patients at an early pre-diabetic stage of disease. While these patients have insulin secretory abnormalities which are less severe than those found in CF patients with diabetes, they are clearly not metabolically normal-should they receive insulin replacement therapy?
It is well known that a decline in lung function and body weight begins 2-6years before the onset of overt diabetes in CF, in the pre-diabetic period. Patients with impaired glucose tolerance have a greater rate of decline in lung function than those with normal glucose tolerance. ${ }^{5}$ Even more concerning is the observation that a very modest elevation of blood glucose to levels $>144 \mathrm{mg} / \mathrm{dl}$ (7.8 $\mathrm{mmol} / \mathrm{l}$ ) leads to abnormally elevated airway glucose concentrations in people with CF, and this is associated with increased bacterial growth. ${ }^{6}$

In this issue of Thorax (see page 574) Suratwala et al report a significant association between lower nocturnal oxygen saturation and worse glycaemic excursion (measured as the AUC during an OGTT) in children with CF. ${ }^{7}$ Remarkably, this cohort consisted of well-nourished children of mean age 14 years with only minimally decreased lung function as demonstrated by spirometry and normal body mass index. This study does not sort out the "chicken and egg' question of whether the stress of increased inflammation and infection resulted in both abnormal glucose excursion and abnormal oxygen saturation, or whether hyperglycaemia per se contributed to lung pathology and oxygen saturation abnormalities. The investigators offer plausible mechanistic explanations for both of these possibilities.

It is also noteworthy that sleep disturbances including obstructive sleep apnoea, a proposed risk factor for insulin resistance and diabetes in the general population, were not more prevalent in the patients with CF and did not correlate with glucose AUC. Interestingly, there were no differences in markers of inflammation between the CF and control groups. This might be explained by the fact that these patients were clinically well without acute illness at the time of testing, and that local alveolar and distal airway inflammation severe enough to result in pulmonary compromise (evident as ventilation-perfusion mismatch) is more difficult to detect systemically. 8 Despite these uncertainties, this study adds to the growing evidence supporting a relationship between respiratory and $\beta$ cell function within the CF population.

Patients with CF have pancreatic exocrine deficiency and low levels of fatsoluble vitamins. In clinical practice it would not be considered ethical to wait until clinical manifestations or complications of these deficiencies were evident before instituting treatment. Digestive enzyme therapy and vitamin replacement 
begin immediately at the time of diagnosis. It is more complicated, uncomfortable and expensive to administer insulin, and the hypoglycaemic effects of insulin overdose are immediately apparent and potentially (albeit rarely) fatal. These factors have resulted in a reluctance to prescribe insulin unless it is 'absolutely necessary'. An accumulating body of evidence suggests, however, that the harmful effects of subclinical insulin deficiency and modest hyperglycaemia may occur very early and have profound consequences in patients with CF, and that current clinical practice may not be sufficiently aggressive. The clinical guidelines committee recently convened by the Cystic Fibrosis Foundation, the American Diabetes Association and the Pediatric Endocrine Society recognised the question of whether to institute diabetes treatment in patients with CF with pre-diabetes as the highest priority research question in this field. ${ }^{9}$

Many of us believe that, by waiting for traditionally-defined diabetes to develop before instituting insulin therapy, we are providing too little, too late to our patients with CF. However, there are still not sufficient data to allow us to ratio- nally determine when the most appropriate time might be. While the study by Suratwala et al does not attempt to answer the question of when glucose tolerance abnormalities reach a 'toxic threshold' in CF necessitating intervention, by demonstrating an association between mild abnormalities in glycaemic excursion and decreased night-time oxygen saturation in young people with CF who were otherwise healthy, these investigators highlight the need for exploration of the very earliest changes associated with insulin deficiency and hyperglycaemia in CF.

\section{Competing interests None.}

Provenance and peer review Commissioned; not externally peer reviewed.

Published Online First 4 May 2011

Thorax 2011;66:555-556.

doi:10.1136/thx.2010.157883

\section{REFERENCES}

1. Schwarzenberg SJ, Thomas W, Olsen TW, et al. Microvascular complications in cystic fibrosis-related diabetes. Diabetes Care 2007;30:1056-61.

2. Moran A, Becker D, Casella SJ, et al; CFRD Consensus Conference Committee. Epidemiology, pathophysiology and prognostic implications of cystic fibrosis-related diabetes: a technical review. Diabetes Care 2010;33:2677-83.

3. Moran A, Dunitz J, Nathan B, et al. Cystic fibrosis related diabetes: current trends in prevalence, incidence and mortality. Diabetes Care 2009;32:1626-31

4. Moran A, Pekow P, Grover P, et al; Cystic Fibrosis Related Diabetes Therapy Study Group. Insulin therapy to improve BMI in cystic fibrosis-related diabetes without fasting hyperglycemia: results of the cystic fibrosis related diabetes therapy trial. Diabetes Care 2009;32:1783-8.

5. Milla CE, Warwick WJ, Moran AM. Trends in pulmonary function in cystic fibrosis patients correlate with the results of oral glucose tolerance test at baseline. Am J Respir Crit Care Med 2001; 162:891-5

6. Brennan AL, Gyi KM, Wood DM, et al. Airway glucose concentrations and effect on growth of respiratory pathogens in cystic fibrosis. J Cyst Fibros 2007;6:101-9.

7. Suratwala D, Chan J, Kelly A, et al. Nocturnal saturation and glucose tolerance in children with cystic fibrosis. Thorax 2011;66:574-8.

8. Ulrich M, Worlitzsch D, Viglio S, et al. Alveolar inflammation in cystic fibrosis. J Cyst Fibros 2010;9:217-27.

9. Moran A, Brunzell C, Cohen RC, et al: CFRD Guidelines Committee. Clinical care guidelines for cystic fibrosis-related diabetes: a position statement for the American Diabetes Association and a clinical practice guideline of the Cystic Fibrosis Foundation, endorsed by the Pediatric Endocrine Society. Diabetes Care 2010;33:2697-708.

\section{The Danish study on cost effectiveness in sleep related breathing disorders - a possible example for Europe}

\section{Ingo Fietze}

Procedures for the diagnosis and therapy of sleep disordered breathing have been well established in healthcare systems for quite some time and effective management of patients with sleep disordered breathing is established in many healthcare settings. ${ }^{1}$ However, there remain many unanswered questions, not only in relation to pathophysiology, prevalence and management but also in relation

Department of Cardiology, Interdisciplinary Center of Sleep Medicine, CCM, Charité-Universitätsmedizin Berlin, Berlin, Germany

Correspondence to Ingo Fietze, Center of Sleep Medicine, Chariteplatz 1, 10098 Berlin, Germany; ingo.fietze@charite.de to cost of the condition and the costeffectiveness of treatments. These questions have come into sharper focus with the current economic pressures. Healthcare payers may accept evidence that treatment reduces comorbidities, costs for physician service and drug treatment, lost working days, stays at hospitals and accidents caused by sleepiness but they could legitimately ask a number of other questions. Which patient has to be treated with which treatment and when should it be started? Are there identifiable patients where a more conservative treatment strategy suffices and others where alternative treatment methods are needed? And do these treatments increase life expectancy and quality of life, and reduce healthcare costs? We have to convince the national reimbursement agencies in every country that sleep medicine does provide cost-effective treatment so that they provide us with sufficient resources to effectively diagnose and treat obstructive sleep disordered breathing.

There is good evidence that untreated sleep apnoea is associated with reduced life expectancy ${ }^{2}$ and high comorbidity. Important comorbid disorders include arterial hypertension, atrial fibrillation, stroke, coronary heart disease, heart failure, diabetes mellitus, atherosclerosis and depression. As a consequence, patients with obstructive sleep disorders consume around $70 \%$ more healthcare resources than matched control patients. ${ }^{3}$ In this issue of Thorax (see page 560), Poul Jennum and Jakob Kjellberg detail the direct and indirect costs in patients with obstructive sleep apnoea (OSA) compared with a control group and patients with snoring or obesity hypoventilation syndrome (OHS). ${ }^{4}$ Patients with sleep apnoea and OHS had more physician visits, were treated more often in outpatient clinics, were hospitalised more, had 